\title{
Potencial Evocado Auditivo de Tronco Encefálico com estímulos clique e Ichirp
}

\author{
Auditory Evoked Brain Stem Potential \\ with click stimuli and Ichirp
}

\section{Potencial Evocado Auditivo de Tronco Encefálico con estímulos clic y Ichirp}

\author{
Barbara Cristina da Silva Rosa* \\ Carla Patricia Cesar* \\ Aline Cabral* \\ Mirelles Santos* \\ Robson Santos*
}

\section{Resumo}

Objetivo: Analisar os traçados do Potencial Evocado Auditivo de Tronco Encefálico com o uso dos estímulos clique e o Ichirp em adultos com audição normal. Método: Trata-se de um estudo transversal, de caráter analítico, quantitativo. Foram analisados exames de Potencial Evocado Auditivo de Tronco Encefálico de 11 adultos ouvintes ( 2 orelhas), na faixa etária entre 20 e 25 anos, sem alterações auditivas. Esses sujeitos realizaram o registro do Potencial Evocado Auditivo de Tronco Encefálico com estímulo clique e Ichirp. O registro das latências e amplitudes da onda $\mathrm{V}$ foi realizado por meio do equipamento Intelligent HearingSystem - IHS, nas intensidades: 80, 60, 40 e 20 dB. Resultados: Não foram observadas diferenças estatisticamente significativas quanto às amplitudes da onda $\mathrm{V}$, quando comparado os registros do Potencial Evocado Auditivo de Tronco Encefálico estímulo clique e do Ichirp, nas intensidades de $80 \mathrm{~dB}(\mathrm{p}=0.11), 60 \mathrm{~dB}(\mathrm{p}=0.14), 40 \mathrm{~dB}(\mathrm{p}=0.96)$ e $20 \mathrm{~dB}(\mathrm{p}=0.21)$. Em relação às latências da onda $\mathrm{V}$, foram encontradas diferenças estatísticas significantes entre os estímulos Ichirp e clique, nas seguintes

* Universidade Federal de Sergipe, Aracaju, Sergipe, Brasil.

Trabalho apresentado no XXIV Congresso Brasileiro de Fonoaudiologia, realizado de 20 a 22 de outubro de 2016, no Centro de Convenções Rebouças, São Paulo, SP, Brasil, como pôster.

Contribuições dos autores:

RFSS e MS - revisão de literatura, elaboração e autorização da versão final; $A C O$ e $C P H A R C$ - revisão e autorização da versão final do artigo; $B C S R$ - orientação em todas as fases da pesquisa, revisão e autorização da versão final do artigo.

E-mail para correspondência: Barbara Cristina da Silva Rosa - centroaudiologiavp@yahoo.com.br Recebido: $22 / 11 / 2017$

Aprovado: 09/03/2018 
intensidades: $60 \mathrm{~dB}(\mathrm{p}=0,003)$ e $40 \mathrm{~dB}(\mathrm{p}=0,016)$. Conclusão: Foram observadas latências maiores para o Potencial Evocado Auditivo de Tronco Encefálico com o estímulo Ichirp; contudo ausência de diferença entre as amplitudes. Houve maior facilidade na marcação da onda V com o estímulo Ichirp.

Palavras-chave: Eletrofisiologia; Potencial Evocado Auditivo; Surdez; Audição; Perda Auditiva.

\section{Abstract}

Purpose: To compare the traces of Brainstem Auditory Evoked Potential with the use of click stimuli and Ichirp in adults with normal hearing. Methods: This is a cross-sectional, analytical, quantitative study. Eleven hearing adults with ages between 20 and 25 years, with normal audiometric thresholds, totaling 22 ears were analyzed. These subjects performed the recording of the Auditory Evoked Potential of Click Brain Trunk and Ichirp. The latency and amplitude of the $\mathrm{V}$ wave was recorded through a computer using the Intelligent Hearing System (IHS) equipment at intensities: 80, 60, 40 and 20 dB. Results: No statistically significant differences were observed in $\mathrm{V}$-wave amplitudes when compared to the Ichirp stimulus with the click, in the intensities of $80 \mathrm{~dB}(\mathrm{p}=0.11), 60 \mathrm{~dB}(\mathrm{p}=0.14), 40 \mathrm{~dB}(\mathrm{p}=0.96)$ and 20 $\mathrm{dB} P=0.21)$. Regarding the latency of the $\mathrm{V}$ wave, significant statistical differences between the Ichirp and click stimuli were found in the following intensities: $60 \mathrm{~dB}(\mathrm{p}=0.003)$ and $40 \mathrm{~dB}(\mathrm{p}=0.016)$. Conclusion: Larger latencies were observed with the Ichirp stimulus.

Keywords: Electrophysiology; Auditory Evoked Potential; Deafness; Hearing; Hearing Loss.

\section{Resumen}

Objetivo: Analizar los trazados del Potencial Evocado Auditivo de Tronco Encefálico con el uso de los estímulos clic y el Ichirp en adultos con audición normal. Método: Se trata de un estudio transversal, de carácter analítico, cuantitativo. Se analizaron exámenes de Potencial Evocado Auditivo de Tronco Encefálico en 11 adultos oyentes ( 2 orejas), en el grupo de edad entre 20 y 25 años, sin alteraciones auditivas. Estos sujetos realizaron el registro del Potencial Evocado Auditivo de Tronco Encefálico con estímulo clic y Ichirp. El registro de las latencias y amplitudes de la onda $\mathrm{V}$ fue realizado por medio del equipo Intelligent Hearing System - IHS, en las intensidades: 80, 60, 40 y 20 dB. Resultados: No se observaron diferencias estadísticamente significativas en cuanto a las amplitudes de la onda $\mathrm{V}$, cuando comparados a los registros del Potencial Evocado Auditivo de Tronco Encefálico estímulo clic y Ichirp, en las intensidades de $80 \mathrm{~dB}(\mathrm{p}=0.11), 60 \mathrm{~dB}(\mathrm{p}=0.14), 40 \mathrm{~dB}(\mathrm{p}=0.96)$ y $20 \mathrm{~dB}(\mathrm{p}=0.21)$. En cuanto a las latencias de la onda $\mathrm{V}$, se encontraron diferencias estadísticas significativas entre los estímulos Ichirp y el clic, en las siguientes intensidades: $60 \mathrm{~dB}(\mathrm{p}=0,003)$ y $40 \mathrm{~dB}(\mathrm{p}=0,016)$. Conclusión: Fueron observadas latencias mayores para el Potencial Evocado Auditivo de Tronco Encefálico con el estímulo Ichirp; sin embargo, ausencia de diferencia entre las amplitudes. Hubo mayor facilidad en la marcación de la onda V con el estímulo Ichirp.

Palabras claves: Electrofisiología; Potencial Evocado Auditivo; Sordera; Audición; Pérdida Auditiva.

\section{Introdução}

A saúde auditiva vem sendo um dos temas mais discutidos no meio científico, pela importância da audição na comunicação humana, sendo imprescindível a identificação precoce das alterações auditivas, a fim de diminuir suas consequências biopsicossociais ${ }^{1}$.

Dentre os vários exames objetivos existentes para o diagnóstico de alterações auditivas, têm-se os Potenciais Evocados Auditivos de Tronco Encefálico (PEATE) que são constantemente utilizados nas avaliações audiológicas de adultos que apresentem zumbidos, perdas auditivas assimétricas ou quando há suspeita de simulação de respostas na audiometria tonal liminar ${ }^{2}$.

O PEATE, além de avaliar a atividade eletrofisiológica do sistema auditivo em resposta a um estímulo acústico, também avalia a função das vias auditivas centrais e periféricas. Os resultados 
obtidos são representados por sete ondas geradas pelo nervo coclear e tronco encefálico, que surgem de zero a oito milissegundos (ms) após a estimulação acústica, em que apenas as ondas I, III e V são analisadas por apresentarem melhores morfologias e amplitudes ${ }^{3,4}$.

O traçado do PEATE é avaliado pela morfologia, latência, amplitude, relação de latência absoluta e interpicos das ondas. Qualquer comprometimento na porção periférica e no tronco encefálico do sistema auditivo compromete o traçado dessas ondas ${ }^{5}$.

O estímulo clique é o mais utilizado na prática clínica por evocar respostas eletrofisiológicas no PEATE, estimular uma região maior da cóclea, produzindo maior sincronia neuronal. No entanto, pesquisas mostram que as respostas ao clique não são totalmente sincronizadas 3,6 .

Quando um clique atinge a membrana basilar, a onda sonora resultante leva um tempo considerável para chegar à base da cóclea, principalmente nas regiões de baixas frequências ${ }^{(7)}$. Sendo assim, o clique é um estímulo de curta duração capaz de excitar a orelha interna na faixa entre $2.000 \mathrm{~Hz}$ e $4.000 \mathrm{~Hz}^{8}$.

Desde a década de 1990, pesquisadores vêm estudando um novo estímulo, denominado de chirp de banda larga, que é um estímulo de ativação neuronal simultânea da cóclea, melhorando assim $\mathrm{o}$ atraso temporal que o estímulo clique ocasiona $\mathrm{e}$ aumentando a amplitude da onda V, principalmente em fracas e médias intensidades. Essa melhora nas respostas acontece, pois no chirp as frequências altas estão atrasadas em relação às frequências mais baixas ${ }^{9,10}$

O chirp diferencia-se do estímulo clique, por causa da maneira como excita a cóclea. O clique é um estímulo de banda larga que foi desenvolvido para que todos os seus componentes de frequências apresente-se de forma simultânea. Dessa forma, ao se considerar a tonotopia coclear, cada região da membrana basilar é estimulada, uma após a outra, da base até o ápice. Com isso, os componentes de frequências baixas levam mais tempo para alcançar o ápice da cóclea, provocando um atraso temporal na excitação de uma parte da membrana basilar. Consequentemente, a ativação das fibras neurais responsáveis pelas regiões basais da cóclea antecede a das fibras apicais em alguns milissegundos. O resultado da estimulação das diferentes fibras neurais em tempos diferentes é a diminuição da sincronia neural necessária para se evocar um potencial auditivo ${ }^{11,12}$.

Existem no mercado diferenças entre os nomes dados ao estímulo chirp, contudo, essa diferença se dá em consequência dos diferentes fabricantes dos equipamentos do PEATE. Vale ressaltar que todo o estímulo da família chirp mantém os princípios básicos do estímulo, no entanto, podem-se observar pequenas alterações que podem interferir na estimulação da cóclea.

Em estudo brasileiro, que objetivou comparar o PEATE com estímulo CE-chirp ao PEATE clique em 12 sujeitos com audição normal, foram comparadas as latências e as amplitudes da onda $\mathrm{V}$ e a presença ou ausência das ondas I, III e V em fortes intensidades. Os resultados mostraram que as latências da onda $\mathrm{V}$, observadas com CE-chirp, foram maiores que as observadas com o clique nas intensidades fracas ( 20 e 40 dB). Já nas intensidades fortes ( 60 e $80 \mathrm{~dB})$, o oposto ocorreu. Maiores amplitudes foram observadas com o CE-chirp em todas as intensidades, exceto em $80 \mathrm{~dB}^{13}$.

Em outra pesquisa foi observado que o PEATE com o estímulo chirp tem, em média, $15 \%$ maior amplitude quando comparada à onda $\mathrm{V}$ do estímulo clique ${ }^{14}$.

Pesquisadores estudaram o PEATE com o estímulo chirp baseado nas latências de bandas derivadas do PEATE em dez adultos com audição normal em dois níveis de intensidade, $50 \mathrm{~dB}$ e 60 dB. Encontraram amplitudes de respostas maiores para o chirp que para o clique e observaram que quanto menor a intensidade, maior a amplitude para o chirp e a latência das respostas se mostraram menores para o chirp que para o clique ${ }^{15}$.

Pesquisa ${ }^{15}$ observou as latências e amplitudes do Potencial de Ação Composto em 16 adultos ouvintes normais, comparando as respostas captadas com os estímulos clique e chirp. Os resultados mostraram que as respostas evocadas por chirp eram melhores do que por clique, visto que a amplitude era maior e a latência menor, o que sugere que as fibras do nervo auditivo respondem com mais sincronia para o chirp.

Cebulla e Elberling ${ }^{16}$ construíram cinco chirps de diferentes durações baseados em diferentes versões de modelos de atraso, denominando-os chirp 1, chirp 2, chirp 3, chirp 4 e chirp 5. Comparando-os com o estímulo clique na avaliação do PEATE em 50 adultos com audição normal nas intensidades de 50 e $30 \mathrm{~dB}$, observaram as ondas III e o complexo 
IV-V claramente em $50 \mathrm{~dB}$, porém na intensidade de $30 \mathrm{~dB}$ esses detalhes tornaram-se menos claros, principalmente para chirps mais longos. Obtiveram respostas com amplitudes maiores e latências menores para o chirp do que para o estímulo clique. $\mathrm{O}$ chirp parece oferecer maior vantagem que o clique nas intensidades mais baixas.

Elberling e Don ${ }^{17}$ sugeriram que os estímulos chirp anteriormente desenvolvidos eram difíceis de serem aplicados de forma adequada, uma vez que dependiam da influência de diferentes mecanismos. Dessa forma, desenvolveram chirps de frequência específica, elaborados a partir das latências do PEATE. Este novo modelo forneceu estimativas do atraso de cada frequência que o chirp deveria compensar. Compararam as respostas de PEATE com o estímulo CE-Chirp e o estímulo chirp nas frequências de 500, 1000, 2000 e $4000 \mathrm{~Hz}$ em 25 adultos com audição normal e em seis níveis de estimulação $(10,20,30,40,60,80 \mathrm{~dB})$. Os autores concluíram que esse estímulo chirp seria o mais eficiente para a gravação do PEATE que posteriormente ficou conhecido como Level Specific Chirp (LS-Chirp).

A literatura evidenciou que o estímulo chirp tem apresentado resultados significantes em adultos e neonatos com audição normal e com perda auditiva. Contudo, percebe-se a falta de pesquisas envolvendo o estímulo Ichirp, tornando imprescindível o estudo desse novo estímulo na prática clínica e no diagnóstico audiológico, visto que alguns equipamentos só dispõem do estímulo Ichirp para evocação de respostas.

Foi verificada, na literatura, apenas uma pesquisa referente ao estímulo I-chirp, realizada em $2016^{18}$, em que foram avaliados trinta neonatos a termo com os estímulos clique e Ichirp em intensidades de $60 \mathrm{~dB}, 40 \mathrm{~dB}$ e $20 \mathrm{~dB}$. Obteve como resultados latências e amplitudes maiores com o estímulo Ichirp quando comparados ao estímulo clique, em todas as frequências testadas. Concluiu que o Ichirp é recomendável para avaliação audiológica infantil, por apresentar boa amplitude da onda $\mathrm{V}$, facilitando a identificação no traçado, além de apresentar latências maiores.

Desta forma, e considerando a evolução constante de novos estímulos do potencial evocado auditivo nos equipamentos audiológicos utilizados, há de se considerar e refletir sobre as normativas e a base técnica científica para a execução de uma prática profissional em audiologia fidedigna.
Sendo assim, o objetivo deste trabalho foi analisar os traçados do Potencial Evocado Auditivo de Tronco Encefálico com o uso dos estímulos clique e o Ichirp em adultos com audição normal.

\section{Método}

Esta pesquisa é de natureza transversal, de caráter analítico e quantitativo. A pesquisa respeitou a Resolução № 466/12, sendo aprovada pelo Comitê de Ética em Pesquisa (CAAE 55350316.0.0000.5546). Esta pesquisa está inserida no Programa de Estudos de Graduação em Fonoaudiologia da Universidade Federal de Sergipe, Campus Prof. Antônio Garcia Filho, no ambulatório de audiologia, mesmo local onde foi realizada a investigação. Todos os indivíduos avaliados na pesquisa assinaram o Termo de Consentimento Livre e Esclarecido (TLCE).

Foram avaliados onze adultos com audição normal (22 orelhas), com faixa etária entre 20 e 25 anos. Todos os participantes realizaram a triagem auditiva em que foram realizados os procedimentos: entrevista contendo identificação, histórico de saúde e queixas audiológicas e inspeção visual do meato acústico externo (MAE) com uso do otoscópio, para verificar a integridade do MAE como também da membrana timpânica. A imitânciometria foi realizada para avaliar a integridade da orelha média e da membrana timpânica, com uso do equipamento AT 235 da Interacoustics. A audiometria foi feita para avaliar o sistema auditivo e o limiar auditivo do sujeito, com o audiômetro AC- 33 da Interacoustics, utilizando-se fones supra-aurais.

Considerou-se como critérios de exclusão a presença de perda auditiva, alterações neurológicas, visuais, psíquicas, má formação de cabeça e pescoço, além de síndromes de qualquer natureza.

$\mathrm{Na}$ avaliação do PEATE, realizada com o equipamento Intelligent HearingSystem - IHS, foi solicitado ao sujeito que ficasse deitado na maca em repouso. Realizou-se a higienização da pele com pasta abrasiva Nuprep ${ }^{\circledR}$, e foram afixados os eletrodos descartáveis de $\mathrm{Ag} / \mathrm{AgCl}$ da marca Meditrace $^{T M} 200$, dispostos da seguinte maneira: eletrodos de referência nas mastóides direita (A) e esquerda (B); eletrodos ativo em região Fz e terra em FPz. Os estímulos clique ou Ichirp foram promediados por meio de fone de inserção ER- $3^{\mathrm{a}} \mathrm{em}$ todos os sujeitos avaliados, sendo que para 5 indivíduos o registro do PEATE foi realizado primeiro 
com estímulo clique e, em seguida, para o Ichirp e para os outros 6 sujeitos iniciou-se com estímulos Ichirp. Foram utilizados os seguintes parâmetros de estimulação e captação: frequência do estimulo: 2000 - $4.000 \mathrm{~Hz}$; taxa de apresentação: 27.7/seg; número de estímulos: 1024 estímulos; polaridade alternada; intensidade: $80,60,40$ e 20 dBnNA; filtro: 100-3000 Hz; janela de análise: de 0 à 24 ms; impedância dos eletrodos: 1 a $3 \mathrm{k} \Omega$. As ondas foram replicadas duas vezes para cada registro.

Os exames foram analisados qualitativa e quantitativamente por duas juízas especialistas e experientes na área de eletrofisiologia da audição. Foram demarcadas as ondas V do PEATE e analisados parâmetros de latência e amplitude para as diferentes intensidades de estimulo (80, 60, $40 \mathrm{e}$ $20 \mathrm{~dB}$ ).

Os dados foram tabulados e processados pelo aplicativo para microcomputador Statistical Package for Social Sciences (SPSS), versão 21.0. Foram aplicadas técnicas de estatística descritiva, e os resultados expressos na forma de tabelas e gráficos. A normalidade das amostras foi observada por meio do teste de Kolmogorov-Smirnov. Para detectar diferenças dos parâmetros de latência e amplitude dos PEATE's registrados por meio do estímulo clique e do Ichirp, foi aplicado o teste não paramétrico de Wilcoxon. Os valores de alfa foram considerados significativos quando menores que 0,05 . O valor de beta estabelecido foi de 0,1 .

\section{Resultados}

A amostra foi composta por onze sujeitos adultos ( 22 orelhas), sendo 9 mulheres e 2 homens, com faixa etária entre 20 e 25 anos, sem alterações auditivas.

Não foram verificadas diferenças para os parâmetros latência e amplitude da onda V do PEATE clique e PEATE Ichirp, entre as orelhas direita e esquerda e, entre os sexos, para valores de $p \leq 0,005$.

Realizou-se a análise dos valores médios de latência e amplitude da onda $\mathrm{V}$ do PEATE clique e do PEATE Ichirp, para diferentes intensidades de estimulação, conforme pode ser visualizado nas Tabelas 1 e 2 .

Tabela 1. P valores da análise da latência da onda $\mathrm{V}$ do PEATE, para os estímulos clique e $I$-chirp, em diferentes intensidades $(\mathrm{N}=22)$.

\begin{tabular}{|c|c|c|c|c|c|c|c|c|}
\hline \multirow{2}{*}{$\begin{array}{l}\text { Latências } \\
\text { absolutas }\end{array}$} & \multicolumn{2}{|c|}{$80 \mathrm{~dB}$} & \multicolumn{2}{|c|}{$60 \mathrm{~dB}$} & \multicolumn{2}{|c|}{$40 \mathrm{~dB}$} & \multicolumn{2}{|c|}{$20 \mathrm{~dB}$} \\
\hline & Ichirp & clique & Ichirp & clique & Ichirp & clique & Ichirp & clique \\
\hline $\begin{array}{l}\text { Média } \\
\pm D P^{*}\end{array}$ & $\begin{array}{c}8,49 \\
\pm 0,43\end{array}$ & $\begin{array}{c}5,61 \\
\pm 0,20\end{array}$ & $\begin{array}{c}9,03 \\
\pm 0,41\end{array}$ & $\begin{array}{c}6,11 \\
\pm 0,28\end{array}$ & $\begin{array}{l}10,04 \\
\pm 0,50\end{array}$ & $\begin{array}{c}6,80 \\
\pm 0,63\end{array}$ & $\begin{array}{c}11,14 \\
\pm 0,73\end{array}$ & $\begin{array}{c}7,67 \\
\pm 0,75\end{array}$ \\
\hline Mediana & 8,53 & 5,63 & 9,05 & 6,10 & 9,95 & 6,78 & 11,05 & 7,63 \\
\hline P Valor & \multicolumn{2}{|c|}{0,11} & \multicolumn{2}{|c|}{0,003} & \multicolumn{2}{|c|}{0,016} & \multicolumn{2}{|c|}{0,098} \\
\hline
\end{tabular}

Legenda: *DP - desvio padrão.

Tabela 2. $P$ valores da análise da amplitude da onda $V$ do PEATE, para estímulos clique e Ichirp, em diferentes intensidades $(n=22)$.

\begin{tabular}{ccccccccc}
\hline \multirow{2}{*}{ Amplitude } & \multicolumn{2}{c}{$\mathbf{8 0} \mathbf{~ d B}$} & \multicolumn{2}{c}{$\mathbf{6 0} \mathbf{~ d B}$} & \multicolumn{2}{c}{$\mathbf{4 0} \mathbf{~ d B}$} & \multicolumn{2}{c}{$\mathbf{2 0 ~ d B}$} \\
\cline { 2 - 9 } & Ichirp & clique & Ichirp & clique & Ichirp & clique & Ichirp & clique \\
\hline Média & 0,998 & 0,850 & 0,848 & 0,647 & 0,768 & 0,544 & 0,523 & 0,430 \\
IDP & $\pm 0,548$ & $\pm 0,372$ & $\pm 0,594$ & $\pm 0,253$ & $\pm 0,540$ & $\pm 0,221$ & $\pm 0,260$ & $\pm 0,150$ \\
Mediana & 0,740 & 0,710 & 0,690 & 0,590 & 0,600 & 0,490 & 0,470 & 0,380 \\
P Valor & \multicolumn{2}{c}{0,11} & \multicolumn{2}{c}{0,14} & \multicolumn{2}{c}{0,96} & & 0,21 \\
\hline
\end{tabular}

Legenda: DP - desvio padrão; Valores de p obtidos por meio do teste de Wilcoxon.

Observaram-se menores valores de latência para a onda V do PEATE clique; contudo ondas com maiores amplitudes para o PEATE Ichirp. Ao aplicar o teste de Wilcoxon, foram constatadas diferenças significativas entre os estímulos Ichirp e clique apenas para a variável latência nos registros do PEATE nas intensidades de 60 e $40 \mathrm{~dB}$ (Tabelas 1 e 2). 
Observou-se na clínica que o estímulo Ichirp se mostrou de visualização mais fácil do que o estímulo clique, apresentando melhor morfologia, principalmente nas intensidades mais baixas, como pode ser visualizado na Figura 1 e 2.

Latência da onda V: clique X Ichirp

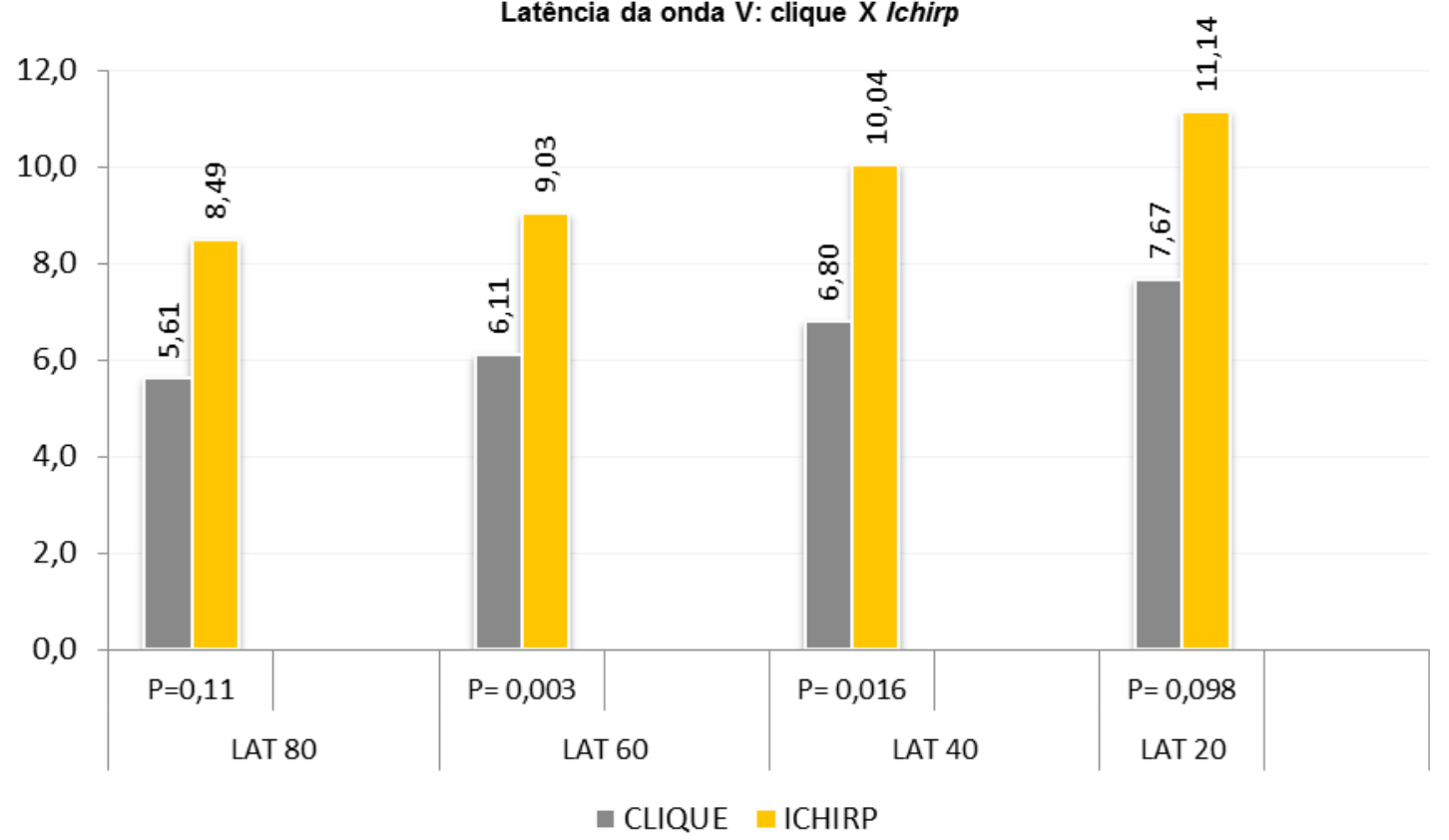

Figura 1. Comparação das latências da onda $V$ com os estímulos clique e Ichirp.
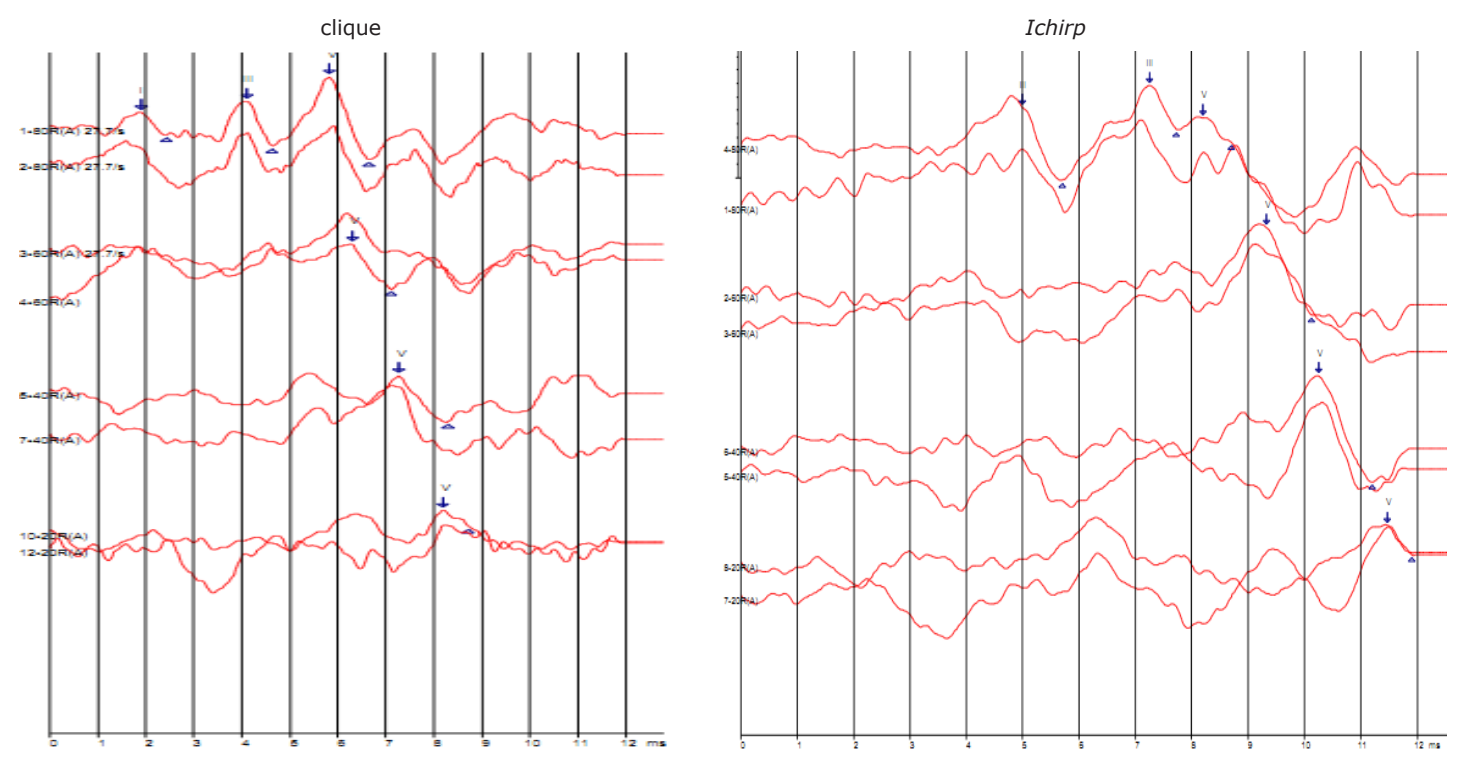

Figura 2. Morfologia da onda V para diferentes intensidades com os estímulos Ichirp e clique. 


\section{Discussão}

$\mathrm{O}$ aumento de pesquisas quanto aos estímulos utilizados para evocar respostas no Potencial Evocado Auditivo demonstra a preocupação de pesquisadores em criar um estímulo que evoque respostas eletrofisiólogicas de fácil visualização do traçado e, consequentemente, diminuição do tempo de realização do exame, facilitando assim, sua utilização na prática clínica. Entretanto, há uma grande quantidade de estímulos para se utilizar no PEATE, em que a maioria desses estímulos foi pouco testada cientificamente o que pode acarretar em sérios problemas na prática clínica, tornando o exame pouco fidedigno $9,10,15,16,18$.

$\mathrm{Na}$ presente investigação foi evidenciado que em todas as intensidades, foram encontradas com o estímulo Ichirp latência da onda $\mathrm{V}$ mais longas que as observadas com o estímulo clique. Isso pode ser observado em pesquisa em que os resultados mostraram que as latências da onda $\mathrm{V}$ observadas com CE-chirp foram maiores que as observadas com o clique nas intensidades fracas $(20 \mathrm{~dB}$ e 40 $\mathrm{dB})$. Achados semelhantes foram encontrados em outros estudos com neonatos, que demonstrou que o estímulo Ichirp apresentou latências maiores em todas as intensidades testadas ${ }^{7,18}$.

No entanto, pesquisa brasileira que comparou os estímulos CE-chirp e o clique, com o PEATE em 20 neonatos com audição normal, concluíram que as latências absolutas da onda $\mathrm{V}$ foram maiores para o chirp em intensidades mais altas enquanto que nas intensidades mais fracas o oposto ocorreu ${ }^{18}$.

As amplitudes observadas com o estímulo Ichirp não foram significativamente maiores que as observadas com o estímulo clique. Este resultado difere dos estudos anteriores que compararam os estímulos chirp ao clique no registro do PEATE, observando amplitudes maiores em todas as intensidades para o estímulo chirp quando comparadas ao clique ${ }^{10,11,15}$.

Nota-se nesta investigação que o estímulo Ichirp é promissor para sua utilização na prática clínica, ademais há poucas evidências científicas que comprove sua eficácia, já que se utilizou uma pequena amostra de adultos jovens, sendo imprescindível pesquisa com outras populações como idosos, neonatos e adolescentes.

Os resultados demonstrados nesta pesquisa contribuem, preliminarmente, para o aprimoramento desse estímulo, verificando suas especificidades e sua aplicabilidade clínica.

\section{Conclusão}

O estímulo Ichirp não apresentou diferenças estatísticas significativas em comparação ao estimulo clique, para as amplitudes da onda $\mathrm{V}$ nas frequências de $80 \mathrm{~dB}, 60 \mathrm{~dB}, 40 \mathrm{~dB}$ e $20 \mathrm{~dB}$. Mas em relação às latências foram encontradas diferenças estatisticamente significativas, sendo maiores para o estímulo Ichirp, principalmente na intensidade de $60 \mathrm{~dB}$.

Entretanto, observou-se melhor morfologia da onda $\mathrm{V}$ para o estímulo Ichirp, principalmente em intensidades mais baixas, facilitando sua identificação nos traçados do PEATE.

\section{Referências bibliográficas}

1. Joint Committee of Infant Hearing (JCHI). Position statement: principles and guidelines for early hearing detection and intervention programs. Pediatrics. 2007; 120: 898-921.

2. Fernandes LCBC, Gil D, Maria SLM, Azevedo MF. Potencial Evocado Auditivo de Tronco Encefálico por via óssea em indivíduos com perda auditiva sensorioneural. Rev. CEFAC. 2013; 15 (3): 538-45.

3. Matas CG, Magliaro FCL. . Introdução aos potenciais evocados auditivos. In: Bevilacqua MC, Martinez MAN, Balen AS, Pupo AC, Reis ACMB, Frota S. Tratado de Audiologia. 2014; 181-195.

4. Grasel G, Ramos HF, Beck RMO, Almeida ER, Sih T. "Audição da perda auditiva na infância- atualização" Revista portuguesa de otorrinolaringologia e cirurgia cérvico- facial; dezembro 2012; 50 (4).

5. Figueiredo MS, Júnior NP. Potenciais Evocados Auditivos de Tronco Encefálico e Emissões otoacústicas. São Paulo: Editora Pulso. 2003; 85-97.

6. Rosa LAC, Suzuki MR, Angrisani RG, Azevedo MF. Potencial Evocado Auditivo de Tronco Encefálico: valores de referência em relação à idade. São Paulo. CoDAS. 2014; 26(2).

7. Dau T. et al. Auditory brainstem responses with optimized chirp signals. Compensating basilar - membrane dispersion.J. Acoust. Soc. Am. Lancaster, Mar 2000; 107(3): 1530-40.

8. Romão MW. Sistema para detecção de limiar auditivo fisiológico com base no potencial evocado em regime permanente [Dissertação]. Belo Horizonte: Universidade Federal de Minas Gerais. 2009.

9. Elberling C, Don M. Auditory brainstem responses to a chirp stimuli designed from derived-band latencies in normal-hearing subjects. J. Acoust. Soc. Am.Lancaster, 2008; 124(5): 3022-37.

10. Leone NL. Aplicabilidade do estímulo chirp na avaliação das perdas auditivas de grau severo e profundo [Dissertação]. Bauru: Universidade de São Paulo; 2014. 
11. Dau T, Wegner O, Mellert V, Kollmeier B. Auditory brainstem responses with optimized chirp signals compensating basilar-membrane dispersion. J Acoust Soc Am. 2000; 107(3): 1530-40.

12. Cargnelutti M, Cóser PL, Biaggio EPV. LS CE-Chirp® ${ }^{\circledR}$ vs. Click in the neuroaudiological diagnosis by ABR. Braz J Otorhinolaryngol. 2017 May - Jun; 83(3): 313-17.

13. Rodrigues GRI; Lewis DR. Comparação dos estímulos clique e CE-chirp ${ }^{\circledR}$ no registro do Potencial Evocado Auditivo de Tronco Encefálico. Rev Soc Bras Fonoaudiol. 2012; 17 (4): 412-6.

14. Hekimoglu Y, Ozdamar O, Delgado RE. Chirp and Click Evoked Auditory Steady State Responses. In: Annual International Conference 23, Istanbul, Turkey 2001; 2, 2084-3.
15. Chertoff M,Lichtenhan J, Willis M. Click and Chirp evoked human compound action potential. J Acoust Soc Am,Lancaster, 2010; 127(5): 2992-4.

16. Cebulla M, Elberling C. Auditory brainstem responses evoked by different ch

irps based on different delay models. J. Am acad audiol, Burlington 2010; 21(7) 452-460.

17. Elberling C, Don M. A direct approach for the design stimuli used for the recording of auditory brainstem response. J. Acoust. Soc. Am.Lancaster. 2010; 128(5): 2955-64.

18. Cargnelutti M. Potencial evocado auditivo de tronco encefálico com estímulos Clique e Ichirp em neonatos. Santa Maria/RS. Dissertação [mestrado em distúrbios da comunicação humana] - Universidade Federal de Santa Maria; 2016. 